\title{
Gestationsdiabetes
}

\section{Die Folgen werden oft unterschätzt}

Liebe Leserinnen und Leser,

von Gestationsdiabetes sind etwa 5\% aller Schwangeren in Deutschland betroffen. Die Tendenz ist steigend, da werdende Mütter immer älter werden und öfter übergewichtig sind als früher. Unbehandelt birgt der Schwangerschaftsdiabetes Risiken für Mutter und Kind. Es besteht unter anderem ein erhöhtes Risiko für Fehlgeburten und für ein erhöhtes Geburtsgewicht des Kindes mit Folgekomplikationen. Bei der Mutter kann ein unbehandelter Gestationsdiabetes zu bleibenden Stoffwechselstörungen führen. Trotz dieser möglichen schwerwiegenden Konsequenzen wird geschätzt, dass immer noch $50 \%$ der Fälle von Gestationsdiabetes übersehen werden. Deshalb fordern Experten der Deutschen Diabetes-Gesellschaft (DDG) seit Langem, die Früherkennung durch Blutzuckertests in die Mutterschaftsrichtlinien aufzunehmen. Das könnte Mutter und Kind besser vor den Folgen eines Schwangerschaftsdiabetes schützen.

Doch auch nach der Schwangerschaft sollten sich Schwangerschaftsdiabetikerinnen regelmäßig untersuchen lassen. Die deutsche GestationsdiabetesStudie zeigt, dass mehr als die Hälfte aller Frauen mit Schwangerschaftsdiabetes innerhalb von zehn Jahren danach einen Diabetes Typ 2 entwickeln (Löbner et al. Diabetes 2006;55:792). Bei Frauen, die während der Schwangerschaft Insulin injizieren mussten, steigt das Risiko, innerhalb der ersten drei Jahre nach der Entbindung an Typ-2-Diabetes zu erkranken, sogar auf $61 \%$.

Auch diese Entwicklungen nach einem Schwangerschaftsdiabetes werden oft unterschätzt. Viele Frauen, die während ihrer Schwangerschaft einen Gestationsdiabetes hatten, lassen sich nach Abklingen der Erkrankung nicht weiter untersuchen. Bleibt ein Typ-2-Diabetes jedoch unentdeckt, kann das zu ernsthaften Folgeerkrankungen wie Nierenschädigungen oder einem Herzinfarkt führen. Deshalb sollten sich Frauen nach einem Gestationsdiabetes zur Diabetesvorbeugung beraten und alle drei Jahre auf Typ-2-Diabetes untersuchen lassen.
Frauen, die einen mit Insulin behandelten Gestationsdiabetes hatten, haben ein noch höheres Risiko für einen späteren Typ-2-Diabetes als jene, die ihren Blutzucker mit diätetischen Maßnahmen und Lebenstiländerungen in den Griff bekommen konnten. Für diese Risikopatientinnen hat die Forschergruppe Diabetes der TU München ein besonderes Angebot:

In der PINGUIN*-Studie erhalten diese Frauen präventiv den DPP-4-Inhibitor Vildagliptin. Zusammen mit einer Beratung zu Ernährung, Bewegung und Lebensstil soll das Medikament einen Typ-2-Diabetes verhindern. Vildagliptin ist ein bereits zugelassenes Medikament für die Behandlung bei Typ-2-Diabetes. Zudem gibt es Hinweise, dass die regelmäßige Einnahme des Medikaments dazu führt, dass sich die Insulin produzierenden Zellen der Bauchspeicheldrüse wieder erholen. Die PINGUIN-Studie nimmt noch weitere Teilnehmerinnen auf.

*Postpartale Intervention bei Gestationsdiabetikerinnen unter Insulintherapie

Anette-Gabriele Ziegler

Leiterin des Instituts für Diabetesforschung, Helmholtz Zentrum München, Sprecherin des Kompetenznetzes Diabetes mellitus, Vorstandsmitglied der Deutschen DiabetesGesellschaft (DDG)

Weiter Informationen zur Studie für interessierte Schwangerschaftsdiabetikerinnen oder Ärzte und Ärztinnen, die die PINGUIN-Studie unterstützen möchten, gibt es im Internet unter www.pinguinstudie.de oder bei der

Forschergruppe Diabetes der TU München Leitung: Prof. Anette-Gabriele Ziegler

Kölner Platz 1, 80804 München

Tel. 089 - 30682917

E-Mail: pinguin@Irz.uni-muenchen.de

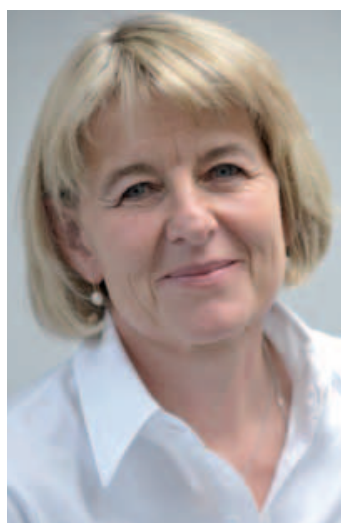

Prof. Dr. med. Anette-Gabriele Ziegler, Leiterin der Forschergruppe Diabetes der TU München 